Hwang, S.-W., \& Roth, W.-M. (2013). The work of lecturing in high school chemistry. Cultural Studies in Science Education, 8, 683-708.

THE WORK OF LECTURING

\title{
The work of lecturing in high school chemistry
}

\begin{abstract}
Lecturing is an important aspect of the culture of science education. Perhaps because of the negative associations constructivist educators make with lecturing, little research has been done concerning the generally invisible aspects of the (embodied, lived) work that is required. Traditional research on science lectures focuses on ideas and (mental) concepts that somehow are "gotten across"; and it is interested in identifying verbal content and visual representations science teachers provide. The purpose of this study is to explicitly describe and theorize the living work of lecturing that produces in a societal arena everything from which students can learn. We use two case studies from the chemistry lectures in a tenth-grade Singapore classroom to exemplify the central role of the performative aspects of lecturing. We articulate and exemplify assertions that (a) corporeal performances differentiate and coordinate the contents of lecturing with its pitch, rhythm, and speech volume, and thereby orient students to specific discourse features of chemistry; and (b) corporeal performances differentiate and coordinate layers of talk with prosody, gestures, and body orientation, and thereby provide students with analogies. We conclude that what is visible in lectures (e.g., scientific discourse, analogies) is always the outcome of the (generally unattended to) corporeal labor including gestures, body orientation, and prosodic features (e.g., shifts in pitch) and that this outcome|labor pair constitutes an appropriate unit of understanding lecturing as societal phenomenon.
\end{abstract}

Keywords: Lecture; Performance; Chemistry; Discourse; Analogy

\section{Introduction}

Lecturing is an important aspect of the culture of science education. Lectures are a common feature in secondary and higher education settings, which suggests that there is something to them that other communicative means - books, the Internet - cannot provide (Friesen 2011). In science education, lectures may actually constitute events in which scientific concepts and thinking come to be performed in the presence of the audience rather than presenting and transmitting concepts as ready-made objects (Roth and Friesen in press). They may thereby assist students in seeing and hearing science concepts in action much like a theater audience witness a drama to play out. But precisely because of their performative aspects, lectures may present concepts with errors, confuse the audience, and render science learning more complex: The narratives lectures provide with graphs may actually contradict the latter (Roth and Bowen 1999a); and the gestures they perform may be temporally shifted with respect to the things in the graph they denote (Roth and Bowen 1999b). In addition to the performance itself, the work of lecturing mobilizes a variety of different but coordinated communicative resources (e.g., "representations"). More recently, the advent of various communication technologies made possible in lecture talk the mobilization of diverse forms of inscriptions (e.g., graphics, movies, and computer simulations). Those resources have transformed lectures from mere "chalk and talk" into multi-modal presentations (Knoblauch 2008) and also have diversified the way by means of which lectures are delivered to the audience. Students not only attend traditional faceto-face lectures in science classroom but also watch online audio/video lectures through various 
technological means. These changes to modern media did not weaken the status of lectures in the school curriculum but increased the bridging role of lectures between verbal communication and technological media (Friesen 2011).

Despite its prevalence, lecturing is one of the less studied areas in science education research. Our recent search of four science education journals in ISI Web of Science (i.e., International Journal of Science Education, Journal of Research in Science Teaching, Research in Science Education, Science Education) shows only 17 research papers published under the topic of "lectures" over the past five years (as of data retrieved on August 8, 2012). Of greater concern than the paucity of studies is the tendency to think about lectures only in terms of their contents that they provide: words and chalkboard inscriptions and the concepts these are said to denote. This tendency is also observable when lectures are thought in terms of presentation slides and media. But if a lecture were only that then what lecturers do would be no more than what they do with a book, text accompanied by images. This raises questions: What more is there to lectures that (perhaps) contributes to their popularity among educators at all levels? What is it that lectures offer over and above verbal content and visual inscriptions on the chalkboard, overhead projector, whiteboard, and the likes? Whereas quite a number of science education studies propose forms of teaching other than lectures and report their effectiveness, it is rare to find studies that explicate what makes lectures more than words plus inscriptions. The purpose of this paper is thus to investigate the performative dimensions of lecturing science (here chemistry). This work tends to be taken for granted and invisible but contributes to the production of this "more" that can be had in lectures but is unavailable in reading a science textbook.

\section{Lecturing: making its work visible}

A study of computer scientists' design work in front of a whiteboard already articulated what the recording medium itself, equivalent to the chalkboard in a lecture, provides. Thus, the board (a) "is a medium for the construction of conceptual objects," (b) "structures mutual orientation to a shared space," (c) organizes talk and writing in a systematic fashion, (d) leads to an arrangement of marks with conceptual ordering between items and a sequential order of their production, (e) constitutes a record of the event, (f) "is a setting for the production and resolution of . . dilemmas," and (g) "is embedded in a network of activities" (Suchman 1990, p. 315-317). What this impressive list does not articulate is the nature of the activities, their performative aspects, or the structure of the work done by the participating scientists on and next to the board.

In this study we are interested in realizing the proposal of "making work visible" (Suchman 1995) to articulate the work of lecturing traditionally considered non-rationalizable, contingent, and embodied and therefore idiosyncratic. Following a conceptualization of the structure of practical action, we understand the phenomenon of lecturing in terms of a couplet (Garfinkel and Sacks 1986) denoted by \{doing [lecturing chemistry]\}. Here, "'doing' designates the work for which the notational particulars," in our study lecturing chemistry, "are its accountable texts" (p. 173). That is, "lecturing chemistry" is a gloss of the events seen on our videotapes; and such a gloss is enabled by certain visual and audible properties of the events. We use the term "gloss" to denote what a knowledgeable person might say when, after opening the door to a presumably unoccupied classroom, she turns around and says "there is a chemistry lecture in there." What such glosses do not describe, however, are the performances themselves. Saying "there is a chemistry lecture in there" does not tell us in what the performance exists, what the participants do to make whatever is happening inside the classroom recognizably a chemistry lecture rather than some other social event. One study, which we emulate here in its method and style, did in 
fact begin to articulate what we are interested in (Garfinkel 2002). Thus, an aspect of the lecture that might be glossed by the term "emphasizing" requires, on the performative side, the production of deictic gestures or changes in prosody (Table 1). Based on existing research on the performative dimensions of lecturing (e.g., Roth 2009), the right column of Table 1 lists the performative equivalents of the glosses marked in the left column. It is these performative dimensions of the lecture that are the focus of this study. That is, we are interested in the how, the work that underlies the performance of chemistry knowledge in a public place rather than the what (content).

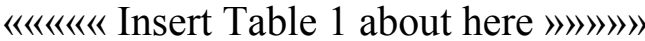

Garfinkel's study (2002) about the work of teaching undergraduate chemistry renders the lecture-specific orderliness that makes lecturing observable in the public place. Because the sociologists involved in the study did not have any chemistry background, they could consider only glossing non-chemistry specific aspects of the performance including $\$$ The lecture hasn’t begun $\ngtr, \Varangle$ Taking and holding places $\ngtr, \nless$ Seeing the room start to fill up $\ngtr, \nless$ Late $\ngtr$, $\Varangle$ Interruption $\ngtr, \Varangle$ Paying attention $\ngtr$, and so on. We use the gloss marks “ $\nless \ldots \ngtr ”$ to set apart what can be pointed out as social facts: but rather than the social facts, our interest lies in the underlying interactive work that allows us to see what the gloss describes. Garfinkel's analyses illustrate the orderly, patterned practice of lecturing as the consequence of the sequential organization of contingent, embodied actions. This is shown in the following example, which became salient for us because we were observing such an event in our videotapes and, after reading Garfinkel's text, were precisely able to locate the performative dimensions of the work involved. In one instance, there is an $\Varangle$ Interruption $\ngtr$. We did not need any special social science method to identify the interruption as interruption. What we initially did not attend to were the performative dimensions that made the event an interruption. In our tapes, the teacher all of a sudden stops in her lecture and walks to the door. There are two students, who had evidently come $\Varangle$ Late $\ngtr$ (another one of Garfinkel's glosses that provided us with a tutorial of locating the work that makes "being late" an observable social fact). The teacher interacts with the two students. They then enter the classroom, take a seat, and the teacher then continues in her lecture. In fact, "being late" and "interruption" both are integral features of the phenomenon of lecture. There is work being performed that gets the students in and the lecture go again. The "tutorial" that assisted us in locating the work (rather than the social fact) reads in this way:

There is an «Interruption $\ngtr$. That is to say, the lecturer finds, and then he brings out the presence of, and then in just so many words he identifies, an interruptionsomeone else's, not his. He begins the enterprise of developing the interruption when he $\Varangle$ Stops lecturing $\ngtr$, (Just how he $\Varangle$ Stops $\ngtr$ we do not know.) Not having video we only notice it after it happens. He walks twenty feet to stand in front of two students who are talking. He speaks to them so that all can hear: "If you two gentlemen are not going to pay attention to the lecture, you may as well leave." Then he returns to the lectern and resumes his lecture. (Garfinkel 2002, p. 230, original emphasis)

To attend to interruptions the lecturer has to stop the ongoing speech and body orientation and take alternatives, which takes up time and physical labor. Recognizable patterned practice of lecturing (i.e., $\Varangle$ dealing with interruptions $\ngtr$ ) is presented to the audience by the embodied 
speaker contingently coping up with incidences in speaking of, pointing out, and orienting toward some aspects of the world. Garfinkel's text in the case of an interruption has become a tutorial for us to find lecturing's work as he suggests that his article consists of a collection of tutorial problems. We understand the text of the findings reported below in the same way, as a tutorial for learning to locate specific phenomena. We return to the idea of the tutorial below.

The emphasis of the performative aspects of social facts, such as getting the lecturing's activity done, highlights the role of the body engaged in contingently speaking of, pointing out, and orienting toward the world. In doing so, the lecturer's body constitutes the expression of the order of the world that it elaborates - " $[\mathrm{t}]$ hought is no internal thing, and does not exist independently of the world and of words" (Merleau-Ponty 1945/1962, p. 213). The study of the orderliness of lecturing brings up the role of the body in indexicality.

Recent educational research on physics lectures provides observations of how a physics professor gets his teaching of scientific concepts done in the public talk (e.g., Roth 2009). These studies analyze the performative aspects (rather than results) of the work of łarticulating a theory $\ngtr, \Varangle$ framing a subject matter $\ngtr$, and $\Varangle$ theorizing a phenomenon $\ngtr$ (see Table 1 ). In these studies, the lecturer's production of sound words, gestures, body positions, body movements, and prosody, which also includes "hesitations, stumbles, mumbles, unfinished sentences, and changes in content and structure" (p. 297), constitutes multiple and incarnate expressions of higher-order communicative unit (i.e., physics concepts). Those studies thereby show the interwoven nature of the body and the world that it inhabits and express in talking physics concepts (Roth 2012b). The findings are also consistent with previous linguistic studies of talking science, which shows that scientists narrate scientific stories and make their way in graphic spaces through multimodal and hybridized discourses (Ochs et al. 1994). For example, when scientists talk about physical events in the presence of visual representations, they produce gestures in direction of the visuals on the board and linguistic expressions that blend physicists and physical worlds (Ochs et al. 1996). Performances interweave the speaking subject (the living body) and the object of thought (the world) in the space of communication that is created simultaneously. In lectures and technology-mediated talk, knowing comes to life much like a drama and emotions come to life in the theater: originally presented in and through bodily performances rather than re-presented by the contents of presentation slides and other audiovisual media.

A very different perspective of the "more" of lectures thus appears when we consider their performative dimensions: The work of lecturing is in the dynamic translation of thinking to communication and again from the latter to the former (Roth, 2010)-lecturing is thinking with hands, eyes, body, and signs (Pozzer-Ardenghi and Roth 2010). In lectures, thought is known through the bodily performance in and for communication (e.g., speech, pointing). Those performances have the potential to bring about a way of perceiving the world differently than before and this changed experience subsequently affects the thought again. Whereas traditional research on lectures focuses on ideas and (mental) concepts that somehow are thought to be "gotten across," materialist approaches are different as they insist all higher psychological functions to exist as societal-material relations. Thus, Vygotsky (1989) proposes that "everything that is internal in higher functions was necessarily once external" (p. 56) and that "external ... means that it was social" (p. 56). Here, the materiality of the societal relation established in and as lecture as well as the performative aspects of communication are at the heart of societyspecific activities. Turning Vygotsky's statement around we might say that if students learn 
anything at all in a chemistry lecture, it has to be a societal relation that subsequently manifests itself as higher-order psychological function on the part of the student.

We emphasize the particular temporal dimensions of lectures. Thus, concepts are not merely gotten across. They do not just suddenly exist out there in the air, as packages to be moved from lecturer to student. Concepts, thought of as a theatrical performance, have some beginning point (like a fertilized egg) and subsequently grow as the lecture moves on (Pozzer-Ardenghi and Roth, in press). The work (labor) of lecturing chemistry consists of the procedure of weaving a societal-material terrain within which some of its constituents such as sound-words, inscriptions, and discursive features of science make metonymic relation to the remaining whole. The "more" of lectures arises from the synergistic effect of this whole network of which the constitutive part represents the whole only one-sidedly (i.e., metonymy).

In what follows, we provide the ethnographic background of data sources and our analytic methods for specifying some of the dynamics of chemistry lecture performances. Then we exemplify our approach and findings in two empirical case examples: First, the corporeal performance differentiates and coordinates the contents of lecture talk with the shift of pitch, rhythm, and volume of speech, and thereby orients students to discourse features of chemistry. Second, the corporeal performance differentiates and coordinates different layers of analogies with prosody, gestures, and body orientation, and thereby provides students with analogies. The two cases each specify and exemplify the work required for teaching discourse features of chemistry in the former and letting the analogous thinking go on in the case of the latter.

\section{Research context}

The purpose of this research is to investigate the work of lecturing that produces, in the social setting, resources for communicating higher-order science concepts. In this study, we analyze empirical data materials collected in a chemistry classroom and examine the analyzability and accountability of lecturing performances. Like studies of mathematicians' lived work of proving mathematical theorems (Livingston 1986) or lecturing (Greiffenhagen in press), which also inspired our study, we investigate the lived work of lecturing chemistry concepts and present the possibility of the pedagogy that articulates lived work of lecturing.

\section{Ethnographic context}

As part of a research project investigating the living procedure of communication and literacy in teaching and learning science, we observed and videotaped a series of chemistry lessons in a 10th grade science classroom in Singapore. The research team worked with a chemistry teacher and one of the classes she taught. In accordance with the research focus on the classroom communication and its performative dimensions, we collaborated with the teacher to plan and carry out chemistry lessons following the school curriculum and academic schedule. The teacher organized her lessons to the lecture-style teaching combined with her competent use of classroom resources (computer, projector, visualizer, and screen). At the time of her joining this study (February 2010) the chemistry teacher had more than ten years of experience of teaching secondary science in public schools in Singapore. During the one-month data collection she taught a class of forty students twice per week and for an hour each. Metals constituted the unit of study and included topics such as physical and chemical properties of metals, alloy, reactivity series, rusting, different extraction methods, and extraction of iron. We observed seven lessons in total: one was held in a chemistry laboratory and the others in the students' classroom. In her lectures she frequently used the whiteboard and occasionally a visualizer to show visual 
graphics in the textbook (e.g., periodic table) or worksheets (e.g., chemistry problems) and a data projector to play video clips from a computer. In this paper, we selected episodes from one lesson focused on the reaction of metals to exemplify the work of lecturing higher-order chemistry concepts. Although we draw our exemplary case materials from one classroom and its teacher and one particular lesson, our results are not limited to her and her class. This is because our analysis is focused on specifying and articulating generalized features of what is being done in the situation, which lets us call what we are observing a science (chemistry) lecture. We analyze all performative features that a lecturer produces in communication and identify interactional resources transcending the individual and that are inherently cultural-historical and ideological. (See subsection on the tutorial.)

Creating data sources

To create data sources, we set up a pair of video cameras and taped visual and sound information the teacher made available in her talk the students. The primary camera was placed at the backside of the classroom to follow the teacher moving about in the classroom while talking to her students (see Figure 1). The other camera functioned as a backup. It was set up at the front end of the classroom near the teacher and the whiteboard; and it was oriented towards students. This allowed us to record students while questioning and answering to the teacher. We used a wireless microphone for the main camcorder (located at the back) to get a clearer recording of the teacher talk as she moved about the classroom as a whole. During the transcription of the data the two camcorders supplied two independent audio tracks of the same classroom conversation and this helped filling up any loss of audibility that happened in one of the two. Part-time and full-time research assistants participated in creating, organizing, and transcribing the data sources. The lessons were filmed in digital $6 \mathrm{~mm}$ tapes and exported to mov format with an accuracy of 40 milliseconds (25 frames/second) at the image level for the subsequent analysis. Audio data was extracted to aif format for the detailed analysis of speech. The speech was initially transcribed in verbatim fashions using conversation-analytic conventions; the soundtrack was analyzed with the PRAAT software designed for linguists to analyze speech intensity, pitch, speech rates, and pauses.

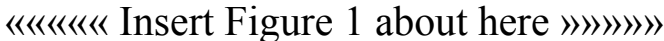

\section{Data analysis}

We combine traditional studies of conceptual content with the analysis of embodied knowing and learning that we have been doing in the previous studies (e.g., Hwang and Roth 2011). In chemistry lessons, chemical discourse consists of different types of signs including words/language, formulas, and models, which have been established and developed at the cultural-historical level over a long period of time (Roth and Friesen in press). For example, $\mathrm{H}_{2} \mathrm{O}$ and "water" were initially two things and correlated to a concept arbitrarily. The two denote the same thing and therefore are synonymous in some ways but not in another because the former co-articulates the chemical composition and structure of the latter. It is only in the 19th century - after the success of decomposing water by electrolysis - that scientists came to correlate water to a combination of hydrogen $(\mathrm{H})$ and oxygen $(\mathrm{O})$, the two elements that had been already known but not yet related to water. In chemistry lessons, this arbitrary correlation has to be made available to individual students who at their levels of development may encounter the formula $\mathrm{H}_{2} \mathrm{O}$ as an alien expression that is seeable and hearable mainly in chemistry lectures. 
In this case, the analysis would be focused on the work of linking the two, that is, what is being done (bodily performances) in lectures to bring two forms of representations together. Therefore, in this paper, we analyze multiple modalities of the bodily expression and the bodily work of making cohere those multiple forms in which signs appear materially.

In our intensive data analyses, the two authors reviewed data sources individually and collectively. We went through the whole data sources until we identified a set of episodes that featured different instances of lecturing and lecture performances-because of space limitations, we present only some of them in this manuscript. We watched the videos while repeatedly stopping, playing, and replaying images frame-by-frame in order to articulate what constitutes visible aspect of lecture talk among interaction participants and what observable communication resources make this possible. We developed our descriptions of lecture events to the microgenetic level at which participants express themselves and perceive things by orienting their bodies, gazing, gesturing, and moving hands/bodies - i.e., "[t]hought and expression, then, are simultaneously constituted" (Merleau-Ponty 1945). The motive for this in-depth analysis is to articulate the real work of lecturing, which is more than a prescription of teaching method that is like a recipe in a cookbook but not equivalent to the work itself. That is, this study specifies the work of lecturing that is not usually specified in method books and courses on teaching chemistry.

The tutorial

In the introductory section, we point to the role of the tutorial in writing a text that makes us attend to, recognize, talk about, and teach others the performative dimensions of social facts. To describe and explicate the nature of a "tutorial," we provide the following example (Roth 2013). Around 2002, one of the authors (Roth) worked with Ken Tobin on a research project in Philadelphia. While driving to the university, Roth talks to Tobin about the listener's contribution to the interactional achievement of conversations, including head nods, body movements, rhythmic gestures, and so on. When the two enter the building, they pass a location where students gathered to talk and work. Roth talks to Tobin, who had been unaware of this phenomenon before, pointing out features that allow the listener's communicative contributions to be recognized. In the same building, the two come across other collections of people in conversation, and now Tobin points to the contributions of the listeners to the joint achievement of conversations. Later, Tobin makes similar remarks in very different social situations away from the university. Whatever Roth had done and said, it was sufficient for Tobin to identify a particular type of collective work. There were no special methods articulated: just how and what humans do in every this situation to pull a conversation of. We understand the text in the next section in the same way. Whatever we describe and explicate, such as $\Varangle$ Orienting students to discourse feature $\ngtr$, our text is to be a tutorial to identify the work required in the collective achievement of the bracketed social fact. Whereas existing research can be understood in the same way, such research also identifies specific research methods that are required for isolating the social fact. Here, as in the Tobin-Roth example, only our everyday "indigenous" methods themselves are required rather than special methods specific to the research enterprise.

\section{The work of lecturing: case studies}

The talk in lecturing chemistry consists not only of words but also of a variety of embodied acts. This talk has a double function: First, it constitutes the means of providing new contents of science and second, it constitutes the terrain of communication in which the new contents mark 
sense to the audience. Lecture talk does not only have contents but also is part of organizing the process of presenting the contents. The two functions mutually constitute each another and drive the constitution of the work of lecturing. For the lecturer, the work of lecturing is to let (a) the ongoing scientific thought unfold by (bodily) engaging in public communication and (b) the ongoing communication move on by (bodily) performing thinking. In this section, we render the procedure of this lecturing's work in two exemplary case analyses of high school chemistry: (a) orienting students to discursive features of chemistry and (b) providing students with analogies. We show that in lectures the chemistry teacher bodily produces and differentiates multiple layers of talk so that in fact there is not a stream of words in lectures but a highly differentiated and layered presentation of performances. Much like Garfinkel tutorial problems, the findings of this section have pedagogical value. Ideally readers would take this article and go into chemistry lectures to then observe first hand the work that this article is attempting to unveil. What we present are tutorials on how to find the work that the reader will be looking at in that case.

Assertion 1. The corporeal performance differentiates and coordinates the contents of lecture talk with the shift of pitch, rhythm, and volume of speech, and thereby orients students to discourse features of chemistry.

Lectures articulate science discourse to be heard on the part of students. This discourse comprises not only science content but also, and importantly so, information about the discourse that provides the audience clues about how to speak/hear the talk. That is, science discourse in lectures comprises both talk and talk about talk (i.e., meta-talk) and therefore is hybridized. For example, a physics lecturer may want to introduce the notion of force scientifically defined in Newtonian mechanics. The lecturer may want to speak the word in specifically defined ways (e.g., force equals the amount of mass multiplied by the acceleration vector) and not want the coming into play of the diverse senses this terminology has in everyday language (e.g., force as energy). For this, the lecturer may want to explicitly tell the audience this difference between scientific and everyday notions of the word or somehow make the scientific notion visible within the science discourse. Yet, for students who are not familiar with the scientific notion of force this discourse may sound strange. Thus, instructors' role in science lectures is to present science discourse visible within this hybridized discourse, which both presents and talks about itself. In the following example, we show how some discursive features are highlighted and made available to students through prosodic properties of speech. Speech rate, intensity, and pitch provide substantial information about different layers of talk-e.g., talk and meta-talk. We analyze the corporeal dimensions of speech and theorize what work makes visible the presence of highlighted discourse features of science.

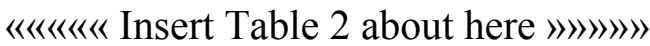

$\Varangle$ Orienting students to discourse features $\ngtr$

In this subsection, we focus on the work employed to orient students to specific discourse features. The shift of pitch, rhythm, and volume of speech constitute the central features of lecture talk that makes it different from textual information (e.g., a textbook). They play a central role in the development of speech contents. In the situation presented in Fragment 1 (see Table 2 ), the chemistry teacher speaks in the presence of grade ten students. The case example exemplifies the work that $\Varangle$ produces talk about a chemical reaction $\ngtr$ and $\Varangle$ coordinates 
representations of a chemical reaction $\ngtr$, which ultimately orients students to discourse features of chemistry. First, one of the ways in which the work is done is by means of a shift in pitch. She says "I say" with a very fast speech rate and then shifts the pitch level up for the three words that she "says" to be saying-i.e., "take over," "replace," and "overtake" (see lines 01-03). She uses the technique of formulating; she says what she is doing (i.e., "I say"). Then she does what she has previously formulated (i.e., named to be doing), and in doing this, her speech shifts into a different pitch level. Second, there is rhythm. She utters three words and they are uttered in the similar lapses of time-i.e., 0.87, 0.80, $0.80 \mathrm{sec}$ each (see Figure 2). The temporal extension of uttering each of the three words is the same for all practical purposes. Also, there is a similar pattern from the first part of each word, then the second part that is much higher (see Figure 2). This is heard as rhythm, and as differentiated from the surrounding talk at the level of "I say." Third, there is a clear split at the volume level between first and second parts of each word, which contributes to the rhythmicity (see Figure 2). The utterance "I say" also has less volume/energy than the average in the three words. This is another distinguishing feature of differentiation (see Figure 2). Fourth, the articulation of the rhythmical utterances co-emerges together with the gestures that also make parallel movements toward different directions (see lines 01-03) and in a regular tempo.

In this example, the teacher does not just produce chemistry content. As an integral part of the lecturing work, she orients students to particular words that are important in the understanding of the chemistry concepts. This orienting does not just happen or exist: it requires work, and the different performative aspects denoted in the preceding paragraph are involved in the performative achievement of the standing out of the important terms. These prosodic features generally are produced without conscious attention. They therefore point us to the function of bodily work in the constitution of scientific concepts.

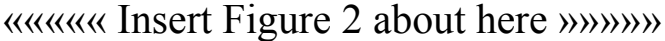

\section{$\Varangle$ Internal structuring}

In this subsection, we describe the work underlying the internal structuring of the performance. Here, the work is done to produce and coordinate the talk at another level with a different focus (i.e., chemical event). She refers to a representation of a chemical reaction on the whiteboard following her utterance "what is happening here now" (lines 04-05). The utterance works as a marker, which is a way of announcing that she is moving into a different domain of talk that would specify the chemical nature of the event. She formulates that something is happening. There is a pause- $0.70 \mathrm{sec}$ (line 05), which contributes to the setting apart of the different levels. She pauses, and thereby sets apart what is coming, and then utters what is happening. She then co-articulates what is happening with the gestural aspect of communication - her left hand stretched to the whiteboard (line 04). She utters "zinc is more reactive than copper" and points out with her right hand " $\mathrm{Zn}$ " and " $\mathrm{Cu}$ " on the whiteboard (lines 06-08). The chemical formula is presented with a marker (lines 04-05) followed by a chemical discourse in the next (lines 06-08). There is work of «internal structuring $\ngtr$ being accomplished in this utterance. First, she presents the word "zinc" (line 06), and then a very brief pause, and the delivery of the relation "is more reactive than" (lines 06-08) in which the "more" is emphasized by the increase of pitch, and then there is a shift on "copper" (see Figure 3 ). What we hear, then, is a comparative, where the two things compared are uttered differently and separated from the comparator "is more reactive than." So again, the chemical content of the 
talk, two metals in the hierarchical list of reaction potentials are of one kind structured within one grammatically full sentence, and the comparison is of another, as the two things in the expression " $\mathrm{Zn}>\mathrm{Cu}$ " are clearly differentiated prosodically. That is, the work of lecturing includes differentiating, and this differentiating is implemented in different ways. A lecture is not just a stream of words but a representationally complex feature that appeals to different forms of consciousness to form a communicative whole. But within this performative whole there is structure; and some of this structure is to produce relations between parts, such as a comparison. That is, a comparison does not just exist but is the result of performative work that requires completion before the comparison as social, that is, observable and reportable fact can exist (e.g., an observer might say, "She made a comparison").

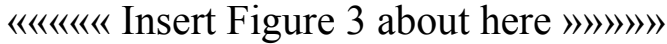

$\Varangle$ Differentiating levels $\ngtr$ and $\Varangle$ coordinating levels $\ngtr$

Chemistry concepts are communicated by means of not only (everyday) language but also representations (inscriptions) distinctive to chemistry. Lecturing chemistry requires discursive movements between different levels of the object-e.g., the discursive level constituted by narratives and the chemical level constituted by chemical inscriptions (e.g., diagrams, graphs, equations). In this subsection, we analyze the lecturer's orientation toward the multiple levels of talk and describe that lecturing involves the work of $\Varangle$ differentiating levels $\ngtr$ and $\Varangle$ coordinating levels $\ngtr$. In the current case example, she is coordinating the discursive and chemical (inscription) levels of talk. This is done a lot with gestures by coordinating her right hand positioned nearer to the board and the left hand nearer to the audience. She talks at one level and also uses gestures to coordinate it with the chemical level. Her right index finger points at the representations on the board: initially $\mathrm{Zn}$ and then $\mathrm{Cu}$ in $\mathrm{CuSO}_{4}$ (lines 06-08). Simultaneously, she rhythmically shakes her left hand in the air when she utters "reactive" (line 07). The gestures she makes can be seen as "beat gestures," and they contribute to marking something like emphasis, or as if she were saying, "listen now." These rhythmical beat gestures are coordinated with the utterance and her eye gaze at the students, and thereby produce an effect of emphasizing some part of the chemical discourse (i.e., "more reactive") and the content of chemical representation (formula) to the audience. She orients toward the chemical representation in the whiteboard on the one hand and toward the audience (students) on the other hand-i.e., double orientation shown by her body oriented toward the students with her right arms stretched back to the whiteboard (see the drawings in lines 06-08). The gestures of the left hand then are coordinated with the utterances and the right index finger pointing out $\mathrm{Zn}$ and smoothly moving to $\mathrm{Cu}$. The marker "we say" (line 10) at first and a part of the chemical discourse ("displaces" in line 11) in the next line are coordinated with the chemical representation. Also, there is a work that provides and structures the talk about chemical reaction. She says "we say" at a lower pitch level and immediately after this, her pitch level is shifted to a different, higher level. The marker and the talk about a chemical reaction are prosodically differentiated. Again, "zinc" and "copper" are differentiated from each other and also from the rest of the words in the sentence.

In drawing students' attention, the language is used not just to talk about something else, but also to talk about talk. This raises questions: How is this possible, this indexicality, this pointing from within communication to a feature of communication? Does this making reference to language fragment have different features from the other kind of discourse, where she only talks about scientific object (i.e., chemical reaction), not the way she talks about it? In written 
language, we use quotation marks or deictic terms such as this or that to point to a feature of language. We are able to mean "this" word or to say he said "this," whereby quotation marks are used to make the word to stand apart as being something different. The analysis of lecturing here is about the bodily resources deployed to make it recognizable so that the speech is about features of the speech itself. What is the form in which this quoting is enacted when there are no "air quotes," which are actually quotation marks taken over from the written language and presented in the form of the index and middle fingers of the two hands drawing ephemeral quotation marks in the air? How does the intonation change so that we recognize which word is highlighted, what a teacher has to say, and what she does not have to say?

First, there is a shift of the agent in this lecture discourse. Previously there was a contrast between the observer, describing person ("I say. . ." in line 01) versus the nature as agent ("What is happening now" in lines 04-05). Thereby a statement of observation was given: zinc is more reactive. Then the discourse comes back to the observer in uttering "we say" (line 10) and "zinc displaces" (lines 10-11). So her work is to lay out and structure the discourse into a part that nature plays, the voice of nature, and another part that the observer plays, the voice of the person. Then there comes a reflexive orientation. She already has laid out nature and observer; and then she orients students explicitly to the discursive feature: "Look at the word I use" (line 14).

Second, she corporeally points to parts of the discourse in the previous levels. The space between the two hands (see the drawing in line 13) develops into one in which discursive features are reflexively oriented. When she turns towards the students, the movements of her two hands (index fingers) produce an iconic gesture resembling "quotation marks." This constitutes a work by means of which certain words stand out. This "air quotes" with index fingers up diminishes to "folded hands" when the contents of the speech change from the word "displace" to other words (i.e., take over, replace, overtake).

Third, she utters "Look at the word I use" and she formulates and then points to a part of the discourse, using in part language "Look" and "at the word I use," and then she does additional pointing using prosody_ "displace" (nature) is differentiated from the previous marker "I use I say" (describing person) by its prosody (jumping pitch from lower to higher) and intensity, and thereby stands out. Then, the differentiation between nature and the describing person continues to be performed prosodically. Whereas the prosodies of "I cannot say" are rather flat and repeated three times, the utterances of "take over," "replace," and "overtake" are made at and across higher or lower levels (lines 15-18).

Fourth, the same features we have identified earlier are also happening here. There are shifts in loudness and speed and pitch that together set the quotation apart. In the current situation, the chemistry teacher says, "I cannot say "replace"" (line 17). Of course she says it; but she says that she cannot say it in another discourse. In fact, then, she is quoting this other discourse, or rather, its inverse, which is the reverse of what she is saying above, where she is quoting herself, "I say." So the case exemplifies multi-layered communication that is occurring and the means of coordinating and differentiating, including the Bakhtinian strategy of direct and indirect speech - utterance takes different forms of direct, indirect, and quasi-direct speech (Vološinov/Bakhtin 1973). Gesture is one of the many means that might be performed to set direct and indirect speech apart from the reporting context (Roth in press). Such gestures are themselves context-dependent so that they have more indexical quality when they are made in reference to an inscription (i.e., in "inscription space") than when they are made in reference to something else not immediately present in the lesson (i.e., "narrative space") (Roth and Lawless 2002). We observe precisely the same in this chemistry class: When the teacher is in inscription 
space she use indexical gestures by referring to something on the board; when she is in narrative space, she uses iconic gestures and prosodies. Therefore, this fragment exemplifies how she coordinates different levels of representation. There are chains of changing agents across the describing person and the nature, which is comparable to a continuum from matter to language (Latour 1999). The world (e.g., the change of the color of the solution observable in the chemistry laboratory) is staged by a human observer and reduced to a specific way of talking (i.e., "displace") among many other alternatives. This referencing put the chemical elements on the stage and amplifies the accountability of the human observation. Much as a drama plays out societal phenomena on the stage (e.g., struggle for power or deception), the teacher performs and brings to life a multitude of distinct things that become part of a continuum only in and through the performance.

Critics of this kind of analysis of performative dimensions often say that it is "only" concerned with social interaction or micro-linguistic features. This example shows how the performative dimension is integral to the distinction between different representations within the chemical discourse and therefore to the conceptual understanding of science. This case example has another feature that shows the social nature of content development. There are additional prosodic means to mark the "lah" (line 20), which is a foreign language talk and rhythmically differentiates words used in everyday context. It is another translation at the heart of the speaking; and it needs to be set apart so that it does not get lost in the stream of speech. A lecture is not just a stream of words or sounds; it is highly differentiated into multiple layers that we may want to distinguish within the performance (see Figure 4). Given that this is not an exclusive characteristic of chemistry lectures but also of everyday language that a lecturer would have recourse to in lectures, the evidence presented in this paper exemplifies both the heterogeneous nature of talking chemistry and the performative nature of conceptions in lectures.

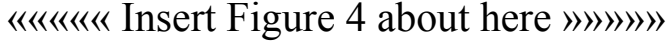

Assertion 2. The corporeal performance differentiates and coordinates different layers of analogies with prosody, gestures, and body orientation, and thereby makes analogies available to students.

Analogies are commonly used in science (chemistry) lectures. Experienced teachers attempt to assist students with analogies in understanding new and unfamiliar contents that are not accessible to direct perception such as microscopic phenomenon at the molecular and atomic levels. An analogy consists of two domains, source and target, between which an analogous relation is made (e.g., everyday and scientific phenomena). Although quite a number of previous science education studies report the significant and productive roles of analogies in learning science and also useful examples of analogies to teach specific concepts (e.g., Duit et al. 2001), they articulate less about what work is required to make visible an analogy as an analogy in lecture talk. In lectures, there is work that is being done to articulate one and the other domain. Also, to articulate the analogous linkage between the two the lecturer does not just say that these are the same but has to physically act to make the analogy exists on the stage, so to speak, so that it can be accessed on the part of the students. In a way, therefore, she says one thing and points simultaneously the other thing. It is this labor of (indexical) linking that makes between the two the analogous relation, and thereby produces an analogy. In this section, we analyze performative 
dimensions and show their structures in the work of linking and therefore making an analogy available to students.

$\Varangle$ Providing an analogy

In Fragment 2 (see Table 3) the teacher is engaged in making an analogy between an everyday social event and a chemical reaction of metal ions. She develops a narrative in which a boy in the class (Wei Xuan) is competing with another for a girlfriend, and thereby points out its analogical relation to the chemical reaction between zinc and copper (II) sulfates. In the following analysis, we approach "providing analogies" differently from what previous researchers have done (e.g., Duit et al. 2001). We are not interested in after-the-fact conceptualization that there is a recognizable analogy that we can observe in science lectures. We do not assume that analogies are simply present by providing an artifact to be used as the source of an analogy. Our question here is: "What is the work (labor) that makes an analogy an objective social fact that members to the setting can point to, argue about, contest, and the likes?" or "What is the work that produces a performance such that an observer can defend saying something like, 'The teacher used an analogy here'?" This is so because the provision of analogies needs to bring about successful communication. We point in this subsection to the work by means of which an analogy comes to be materially available to students rather than simply being given to them like an immaterial gift (e.g. idea) that somehow goes from one mind to another mind. We maintain: It is in and through the lecturer's corporeal work that analogies become accountable, social facts the presence of which students, observers, and researchers can point to.

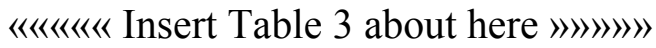

In this situation, the teacher says, "you told me zinc won ..." (line 34), and thereby starts her talk from indirect speech, that is, speech within speech (Vološinov/Bakhtin, 1973). This makes use of the previous discussion and takes it as the taken-for-granted terrain of the talk that follows. Here, the question would be how she sets apart the present level of speech from the indirect quotation, what the students have said? She does it by means of shifts in the prosodic properties of her speech, which is set apart from the previous utterance with a pause $(0.2 \mathrm{sec})$ and increased speech intensity and decreased speech rate after the pause. She then goes on to say, "the chemical world and the real world are very alike" (lines 36-38). She sets up an analogy to come in relation to the displacement reaction between zinc and copper. Yet, this is not the analogy itself but simply a marker that what is to come in fact is an analogy. There is some babble, then she says "all right," which is also a marker that she is beginning now, and there is a pause that sets apart what is coming, and then the beginning of the analogy. Here we see the work of formulating (i.e., what is to come is an analogy between chemical world and real world) and setting apart ("all right," pause [lines 39-40]) the analogy from the remaining talk. All of this is to differentiate the different "channels" of communication (i.e., talk and meta-talk), which makes the conceptual structure of the analogy available to students. In the next lines, there is a lot of setting up. She establishes that Wei Xuan has qualities that make him stand out compared to the other (lines 54-68); and then she says, "what would happen?" (line 71). She marks the analogical situation "exactly like zinc now" (line 73); so Wei Xuan is just like zinc. We see the work of marking the property that is analogical: Wei Xuan is "more handsome" (line 78), "smarter" (line 79), just as zinc is more electronegative. Here (source) and there (target) is a 
competition, and here and there the thing that has more of something that will win. And then she moves again to the chemical context: "exactly the same thing over here" (lines 91-92)

In this situation, we find different levels of talk — chemical element, boyfriend — and the meta-language that mediates between the two but is part of the same talk. To understand an analogy, differentiations need to be made. How is meta-talk performed so that it can be heard as separated from the other two forms of talk? If we just attend to a stream of words, this differentiation cannot be made. We find it in speech properties such as pauses (e.g., $0.8 \mathrm{sec}$ between "the chemical world" and "the real world"; see line 37) and intonation (similar rhythms of speaking the two words). The two parts of the analogy (chemical world, everyday world) are played out in parallel in the same modality (see the horizontal relation in Figure 5). However, because they are evoked in time, they have to be linked across time (horizontal arrow, $\rightarrow$, in Figure 5) rather than, for example, like the verbal expression coincident with pointing to the chemical equation on the board-i.e., verbal and chemical, because in different modalities, can appear simultaneously (vertical arrow $(\uparrow)$ in Figure 5). The coordinating device is the performance of an indexical gesture. In this case of the analogy, both chemical talk and real world talk appear in the verbal modality; but the equivalence needs to be shown in time. In a way, we also have a case of two different levels, one verbal chemical the other verbal everyday life, but they cannot be connected in the same way. So what is the work and what are the devices mobilized to make this coherence, this linking together? She writes an equation $\mathrm{R}+\mathrm{BY}$ and points to each component while talking about "Wei Xuan" (R), "you" (B), and "your girlfriend" (Y). So she actually produces something that will allow another bridging move. She writes the analogy (source) in the form of an equation, using the analogy (source) names, but writes something that has the same structure as the chemical equation. She creates another representation in addition to the story to get a four-part structure of the analogy (see Figure 5). The vertical alignment is done using gesture (see drawings in Table 2), the horizontal alignment in the uppercase is done via words like "exactly like" (line 73) and the lower one is through colocation on the chalkboard, one below the other, and structurally exactly in the same way, that is, iconically ("exactly like" functions like an iconic gesture). Actually, there is another complexity, as she puts the lab observation also on the chalkboard: the words "blue" and "colorless solution" written underneath the chemical equations. And she does point to the chalkboard when she says "exactly the same thing over here" at the end (lines 91-92), that is, by co-locating the verbal real world talk with the chemical equation, that is, she also does a diagonal linking (see the diagonal arrow $(\measuredangle)$ in Figure 5).

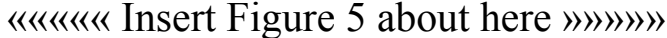

The findings of this section provide a unique perspective of analogy that goes against what people seem to assume in the previous studies. In many studies on analogies, it is assumed that students can see-if they see - two kinds of things, phenomena and representations in relation to each other. But the analysis in this section shows that the analogical relation between the two is not actually something to see or something that needs to be seen in the source object. It is performed. Vygotsky (1989) suggests in his text on concrete human psychology that higherorder psychological functions are external first, physical material relations between people. If there is a conceptual relation, it has to be somehow made available in the social intercourse of people before it appears for the person as something like an analogy. In lectures it is not just like that somehow the stuff is presented and then somehow shows up in the mind. If there are 
relations, then these relations are actually there because of the labor that goes into producing, showing, and exhibiting the relation between the two. This is what we see here in this example. If the lecture does not produce this relation, students are simply presented with two phenomena — one considered analogous to another - and do not see the analogy (e.g., Roth and Duit 2003). So in this example the chemistry teacher actually does a lot of work; and we describe this work by means of which one thing becomes analogous to another. The relation is physically and literally there, where it can be pointed to, because it is performed in words and with the body. The presence of the taken-for-granted work of $\Varangle$ providing an analogy $\ngtr$ becomes visible if we consider a case in which there are interruptions (e.g., arising from the collective work of sleeping (students) and noticing sleeping or coming late and noticing an instant of coming late to the lecture [Garfinkel 2002]) and to the collaborative work of seeing an obstacle, stopping lecturing, fixing the obstacle, seeing the removal of the obstacle, and continuing the lecture. In these situations of breakdown, the lecture would not unfold without the actual work that gets the lecture back on track to produce the phenomenon as a whole - getting the lecture back on track is integral to the work of lecturing. This is so because the teacher needs to actively attend to bringing lectures back into the normal or the context in the normal mode. Analogies cannot be immediately given to students without considerations of the work that the teacher has to do to show how this is like that. This showing is performance.

\section{Conclusions: toward a dialectic theory of lecturing}

The purpose of this study is to investigate the work of lecturing, which is usually taken for granted but in fact contributes to the production of the "what more" of a lecture over a book. Our thorough analysis of the two case examples shows that chemistry lectures make available discursive features of chemistry and analogies through the multiple layers of corporeal performances. Representational resources (e.g., words, chemical equations on the board) do not provide higher-order thinking by themselves but obtain their accountability by becoming part of the performance. Just as we can see hate, love, and jealousy on the theatrical stage, we can see chemical thinking occur in the lecturing performance on the classroom stage. Thus, in those examples, the knowledgeability of the reactivity of metals would refer to competent participation (e.g., seeing/hearing) in the communication that realizes this higher-order thinking with rhythm and pace, sound and melody, speech volume, gestures, and the interlacing of these features. This study shows that the consideration of bodily, performative dimensions of lectures has great potential for the study of everyday, social origins of higher-order thinking (e.g., analogy) and associated, pedagogical principles of lecturing - e.g., to lecture is to bring the scientific objects back to their places of communication within social practice. Because we propose that to lecture is to corporeally perform relations, there are significant implications for teaching and learning science.

First, this study extends the theoretical unit of analysis when we think about the "more" of lectures and their role in the development of higher-order thinking. Higher-order thinking has been one of the central problems in science education. In the past, traditional research investigated knowing and learning as something exclusively occurring in the brain and therefore considered higher order thinking as mental properties. More recently, the field of research has evolved to conceive of conceptions and thinking to involve the body (embodiment literature) or in terms of culture (social constructivism, socioculturalism). The two bodies of literature have contributed to the scholarship by the embodiment literature expanding the research domain of cognition to the role of the body in shaping the mind and by the social constructivism articulating 
the cultural (social) nature of knowing. Yet, there are also numerous problems with these approaches and their combinations. The division between the body and culture maintained by the two research traditions impedes with the theorization of thinking as a material, living, dynamic, and therefore ever-changing process. In this study, we show the dynamic procedure of higherorder thinking at the interface of the body and culture-i.e., in lectures the performance of the body is the body of chemistry concepts (contents). Thus, this study exemplifies a way of integrating the role of inscriptions (e.g., chemical equation) with the (inscribing) work that makes those inscriptions accountable in (classroom) communication. What is visible in lectures (e.g., scientific discourse, analogies) is always the outcome of the (generally unattended to) corporeal labor including gestures, body orientation, and prosodic features. This outcome | labor pair constitutes an appropriate unit for understanding the development of higher-order thinking as both corporeal and social (accountable) phenomenon.

Second, the extended unit of lecturing allows rethinking Vygotsky's concept of the zone of proximal development that has been widely adopted among educators to theorize and explain the development of higher-order thinking (e.g., Shepardson and Britsch 2006). Many researchers take words and representations to analyze the development of thinking when students interact with knowledgeable peers or teachers. This word/representation-centered approach makes it possible to focus on individual speakers and the contents of their speech, and thereby treat the interacting students and teachers as "opposing individuals" (Roth and Radford 2010). However, in doing lectures a lecturer not only talks but also addresses the audiences, which means the audience is already presupposed and constitutive part of the lecture performance. In our case examples, the chemistry teacher's body orientation, gestures, and prosody of speech have been oriented toward the students in the classroom (i.e., narrative space) all the while integrating inscriptions on the board/contents as part of the space of the lecturing work (i.e., inscription space). Thus, the chemistry teacher's body simultaneously facing students and speaking a narrative while pointing out inscriptions on the board (see line 74 in Table 3 for an example) shows her particular orientation to the audience and at the same time the accountability of/for the subject matter. She literally orients toward the accountability of subject matters by referring to what is to be taught and what is this subject matter. She renders or makes visible what is on the board, and thereby takes the accountability to her teaching of the subject matter. At the same time, this orientation is toward students' understanding if we take narrative space as orienting toward more everyday world. Therefore, the body's double orientation provides students conceptual possibilities for experiencing the relation between two independent entities - what is on the board and what is told in the narrative - in the form of social relation. The teacher's body being involved in the narrative space (i.e., the body oriented toward the audience) and simultaneously in the representational space (i.e., her arm placed far back on the board) opens the possibility to be related to the ongoing conceptual thinking (e.g., analogy). In the space of the double orientation the narrative is configured in a particular way in relation to the ongoing lecture accounts and the inscription space is configured in a particular way in relation to the narrative unfolding in mundane, everyday language - "Wei Xuan" as a particular student and "R+BY" written on the board exemplify these configurations. The teacher's body exemplifies that the zone of proximal development is generated by the performance that produces and links the narrative and inscription spaces rather than by words and representations.

Given that many colleges and universities tend to put efforts to design courses for teaching development and to support new and existing professors (e.g., Gallos et al. 2005), this study has implications for the professional development of high school teachers and university instructors 
lecturing science. One of the avenues we pursue is related to Vygotsky's (1989) diction that every higher-order psychological function is a social relation first. If Vygotsky is correct, then (a) preservice teachers may learn to become excellent lecturers by lecturing "at the elbow" of another and (b) just talk is insufficient for the audiences, which we want to see somehow involved in the performance rather than being mere spectators. Much like a theater audience empathizes with the actors, feeling the emotions and tensions that the play is communicating, lectures need to resonate with student audiences to increase their part in the collective production of the lectures. We would then expect that students learn to perform scientific concepts and thinking much like the lecturer does.

This also raises another issue to be addressed in future research, one that is related to the audience. Rather than viewing students as empty vessels on or subjects of the receiving end, the perception of lecturing performances also requires work. What is this work required to hear and see scientific concepts in the bodily performances of the lectures? What is the work required to structure a series of sound-words so that it is heard as talk and meta-talk? What is the work required to hear and see an analogy unfolding before one's eyes? First-person methods may be interesting alternatives to the study of perceptual dimensions of knowing and learning (Roth 2012a). These methods show, for example, how even the perception of a simple line requires work: (a) the eye moving along the line to constitute its longitudinal nature and extension and (b) the eye moving back-and-forth from the line to the perceptual to constitute the presence of the line.

As for the question of what individual science teachers or lecturers can do about their practices of lecturing to make their lectures more effective, we propose that this study can play the role of a tutorial that guides peer observation or video analysis of science lectures in the same way we were guided by Garfinkel's (2002) analysis. In so doing, they come to better understand lecturing in the way a novice cook learns while using a recipe as a tutorial and watching a more expert cook prepare the meal. The effect of such a course of action would be that the novice and teacher would come to develop an understanding of the work required to produce a particular lecture feature. The performative features analyzed in this study constitute exemplary materials that science teachers or instructors can refer to when they engage in teacher research to investigate the procedure of presenting science contents and thinking in and about their own lectures. We propose that the following features are some of the phenomena that are worth looking at and investigating as to the intent to which they constitute foundational moments of lecturing's work: (a) rhythm and pace in communicative performances and across all communicative modalities (e.g., pitch, pitch contour, rate, volume, body position, hand/arm gesture, head movements) both within (as in lectures) and between individuals (as in classroom interactions); (b) sound and melody in communicative performances and across all communicative modalities both within and between individuals; (c) apparent arbitrary movements and sounds that seem to provoke insight and learning; (d) the interlacing of pacing, pitch, and pitch contours in speech (vocal cord movements); (e) the relation of scientific sense and the above features that require in a fundamental manner the living/lived senses; and (f) the relation of thinking and speaking within the "elementary 'cell' that cannot be further analyzed" (Vygotsky 1986, p. 212). The result of such an exercise, therefore, would be an understanding of the actual work rather than a generic description of lecture features that are good or bad.

\section{Acknowledgement}


This work was supported by a research grant from the Office of Education Research, National Institute of Education Singapore (\#OER 7/09 HS). We are grateful to the teacher and students for their interest and participation in this research project. Our thanks are extended to Adrena Binte Adnan Lee Abdullah, Jae Bum Lee, April Sim Pei Yun, and Praveena Raveendran Pillai, who assisted us in the establishment of the database of the research project.

\section{References}

Duit, R., Roth, W.-M., Komorek, M., \& Wilbers, J. (2001). Fostering conceptual change by analogies: between Scylla and Charybdis. Learning and Instruction, 11, 283-303.

Friesen, N. (2011). The lecture as a transmedial pedagogical form: a historical analysis. Educational Researcher, 40, 95-102.

Gallos, M. R., van den Berg, E., \& Treagust, D. F. (2005). The effect of integrated course and faculty development: experiences of a university chemistry department in the Philippines. International Journal of Science Education, 27, 985-1006.

Garfinkel, H. (2002). Ethnomethodology's program: working out Durkheim's aphorism (Edited by A. Rawls). Lanham, MD: Lowman \& Littlefield.

Garfinkel, H., \& Sacks, H. (1986). On formal structures of practical action. In H. Garfinkel (Ed.), Ethnomethodological studies of work (pp. 160-193). London: Routledge \& Kegan Paul.

Greiffenhagen, C. (in press). The materiality of mathematics: Presenting mathematics at the blackboard. British Journal of Sociology.

Hwang, S., \& Roth, W.-M. (2011). Scientific \& mathematic bodies: the interface of culture and mind. Rotterdam: Sense Publishers.

Knoblauch, H. (2008). The performance of knowledge: pointing and knowledge in Powerpoint presentations. Cultural Sociology, 2, 75-97.

Latour, B. (1999). Pandora's hope: essays on the reality of science studies. Cambridge, MA: Harvard University Press.

Merleau-Ponty, M. (1962/1945). Phenomenology of perception (Translated by C. Smith). New York, NY: Routledge.

Ochs, E., Gonzales, P., \& Jacoby, S. (1996). "When I come down I'm in the domain state": grammar and graphic representation in the interpretive activity of physicists. In E. Ochs, E. A. Schegloff, \& S. A. Thompson (Eds.), Interaction and grammar (pp. 328-369). Cambridge: Cambridge University Press.

Ochs, E., Jacoby, S., \& Gonzales, P. (1994). Interpretive journeys: how physicists talk and travel through graphic space. Configurations, 2, 151-171.

Pozzer-Ardenghi, L., \& Roth, W.-M. (2010). Staging and performing scientific concepts: lecturing is thinking with hands, eyes, body and signs. Rotterdam: Sense Publishers.

Pozzer-Ardenghi, L., \& Roth, W.-M. (in press). A cultural-historical perspective on the development of concepts and ideas in science lectures. Cultural Studies of Science Education

Roth, W.-M. (2009). Realizing Vygotsky's program concerning language and thought: tracking knowing (ideas, conceptions, beliefs) in real time. Language and Education, 23, 295-311.

Roth, W.-M. (2010). Thinking and speaking: a dynamic approach. In W.-M. Roth (Ed), ReUniting sociological and psychological perspectives (pp. 113-143). Dordrecht, The Netherlands: Springer-Verlag.

Roth, W.-M. (2012a). First person methods: Towards an empirical phenomenology of experience. Rotterdam, The Netherlands: Sense Publishers. 
Roth, W.-M. (2012b). Tracking the origins of signs in mathematical activity: A material phenomenological approach. In M. Bockarova, M. Danesi, \& R. Núñez (Eds.), Cognitive science and interdisciplinary approaches to mathematical cognition (pp. 182-215). Munich, Germany: LINCOM EUROPA.

Roth, W.-M. (2013). What more in/for science education?: An ethnomethodological perspective. Rotterdam, The Netherlands: Sense Publishers.

Roth, W.-M. (in press). At the intersection of text and talk: On the reproduction and transformation of language in the multilingual evaluation of multilingual texts. Semiotica.

Roth, W.-M. \& Bowen, G. M. (1999). Complexities of graphical representations during lectures: A phenomenological approach. Learning and Instruction, 9, 235-255.

Roth, W.-M., \& Bowen, G. M. (1999). Decalages in talk and gesture: Visual and verbal semiotics of ecology lectures. Linguistics \& Education, 10, 335-358.

Roth, W.-M., \& Duit, R. (2003), Emergence, flexibility, and stabilization of language in a physics classroom. Journal of Research in Science Teaching, 40, 869-897.

Roth, W.-M., \& Friesen, N. (in press). History and the relationship between scientific and pedagogical knowledge: anatomy lectures then and now. Journal of Curriculum Studies.

Roth, W.-M., \& Lawless, D. (2002). When up is down and down is up: Body orientation, proximity and gestures as resources for listeners. Language in Society, 31, 1-28.

Roth, W.-M., \& Radford, L. (2010). Re/thinking the zone of proximal development (symmetrically). Mind, Culture, and Activity, 17, 299-307.

Shepardson, D. P., \& Britsch, S. J. (2006). Zones of interaction: differential access to elementary science discourse. Journal of Research in Science Teaching, 43, 443-466.

Suchman, L. (1990). Representing practice in cognitive science. In M. Lynch \& S. Woolgar (Eds), Representations in scientific practice (pp. 301-321). Cambridge, MA: MIT Press.

Suchman, L. (1995). Making work visible. Communications of the ACM, 38(9), 56-64.

Voloshinov, V. N./Bakhtin, M. (1986/1929). Marxism and the philosophy of language (Translated by L. Matejka \& I. R. Titunik). Cambridge, MA: Harvard University Press.

Vygotsky, L. S. (1989). Concrete human psychology. Soviet Psychology, 27(2), 53-77. 
Table 1. Some possible pairings of glosses and their performative equivalents

\begin{tabular}{ll}
\hline Gloss & Performance \\
\hline Emphasizing $\ngtr$ & Deictic gestures, changes in prosody \\
$\Varangle$ Underlining $\ngtr$ & Descriptions (verbal, iconic gestures) \\
$\Varangle$ Making a link $\ngtr$ & Drawing a chalk line, using a metaphoric gesture for a link \\
$\Varangle$ Differentiating $\ngtr$ & Beat gestures, prosody (pauses, changing rate, intensity, pitch, \\
& contour) \\
$\nless$ Articulating the theory $\ngtr$ & Writing inscriptions (graph curves, equations), eye gaze, body \\
& orientation, hand/arm movement (placing a chalk), gestures \\
& (iconic), prosody \\
& Writing inscriptions (reference lines, variables), eye gaze (at \\
& the inscriptions), body movement (around inscriptions), \\
& prosody \\
& Gestures (iconic, deictic), writing inscriptions (marks on a \\
& graph), prosody
\end{tabular}

Note: Following Garfinkel (2002), ticked brackets $\Varangle \ngtr$ are used to mark the gloss and indicate our intention to find out "the locally produced, naturally accountable lived phenomenon of order" of lecturing. 
Table 2. Transcript of fragment 1

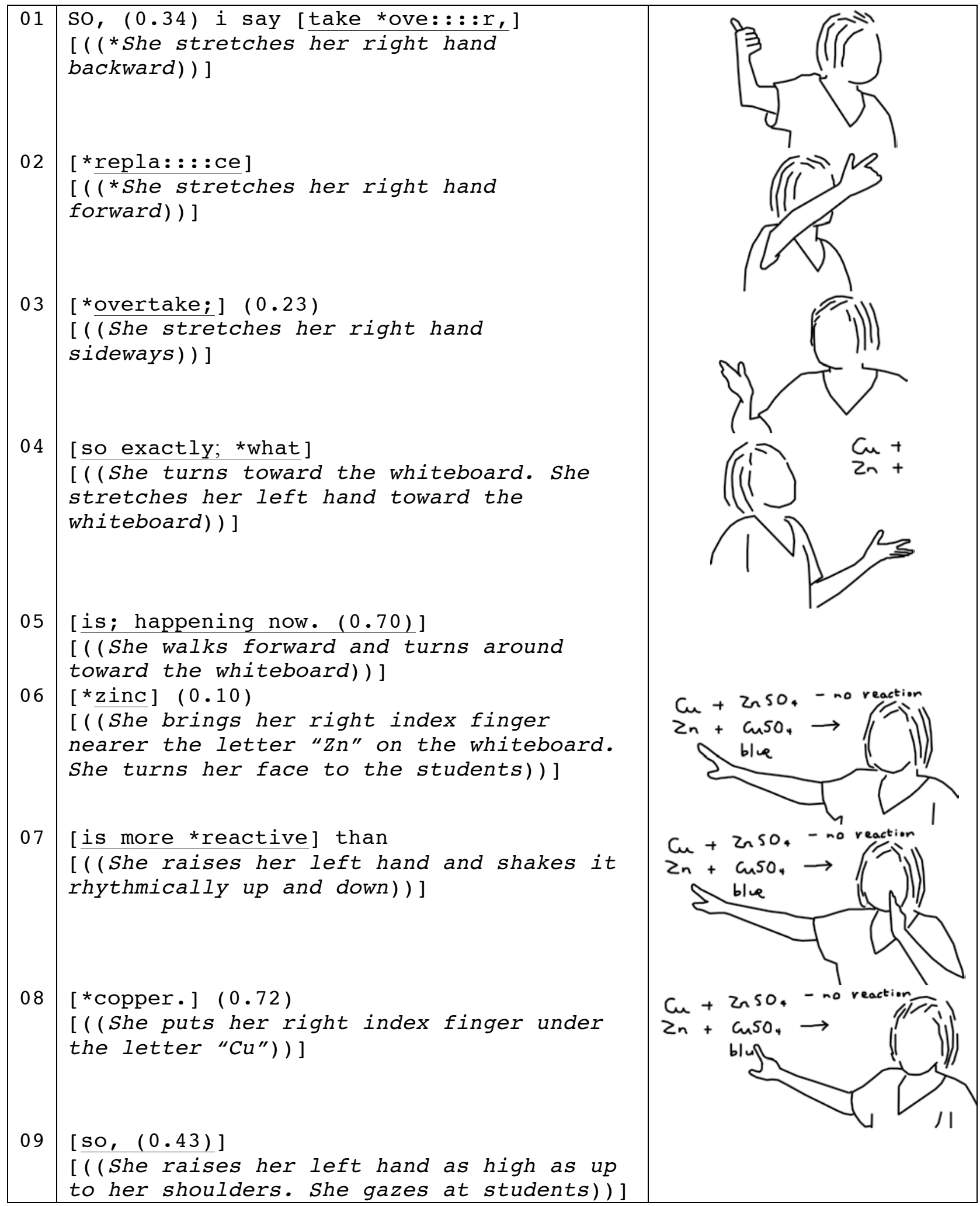




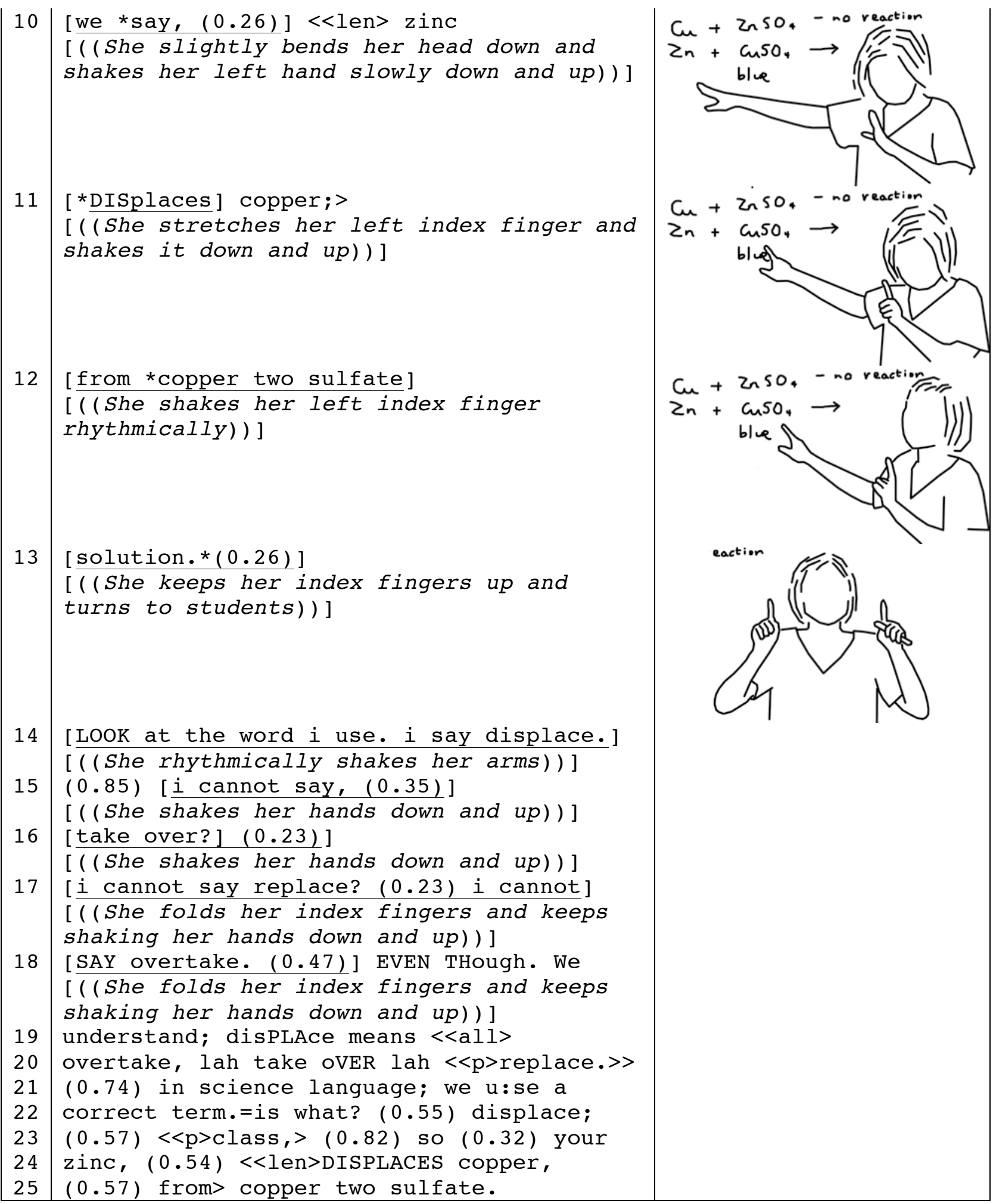


Table 3. Transcript of fragment 2

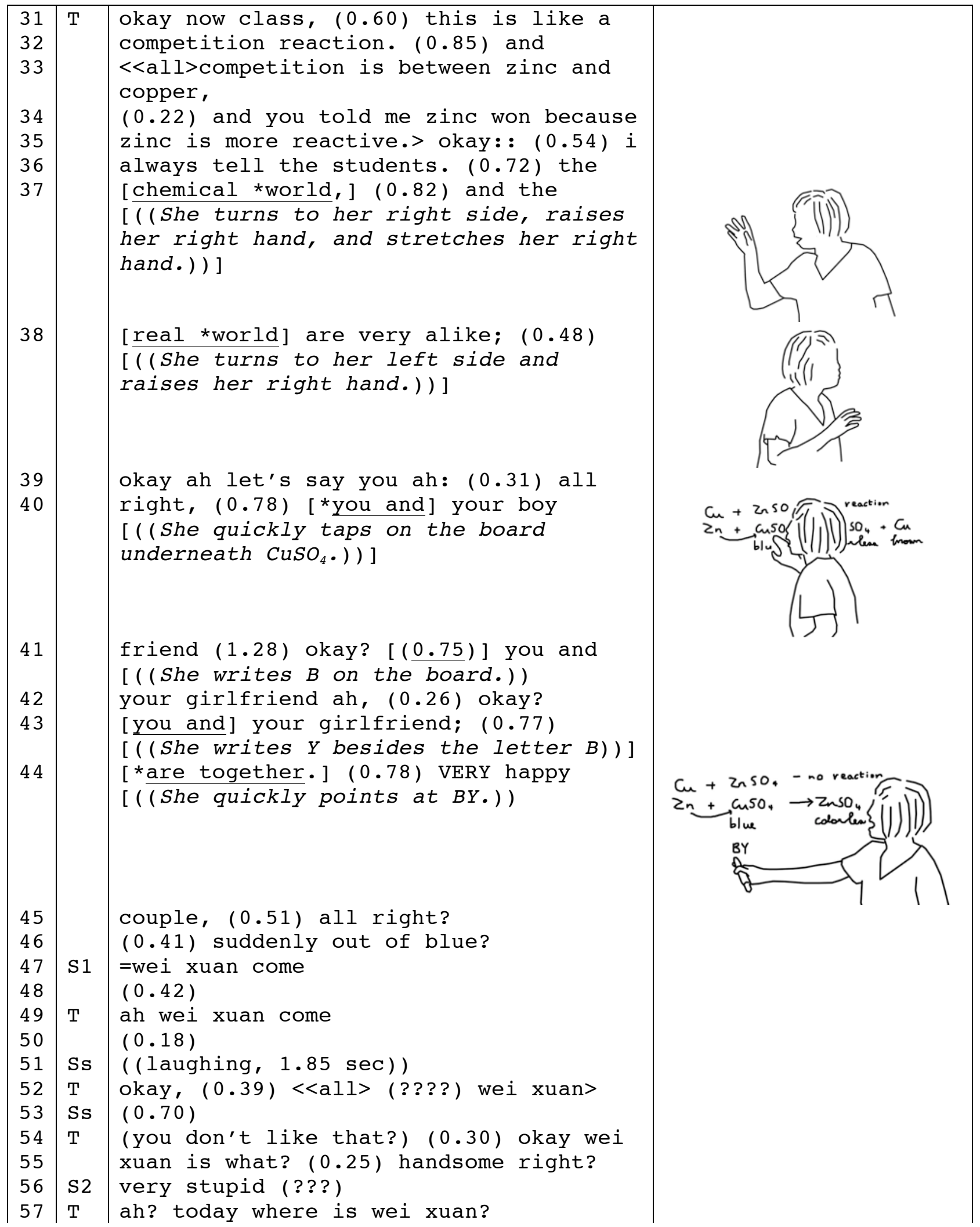




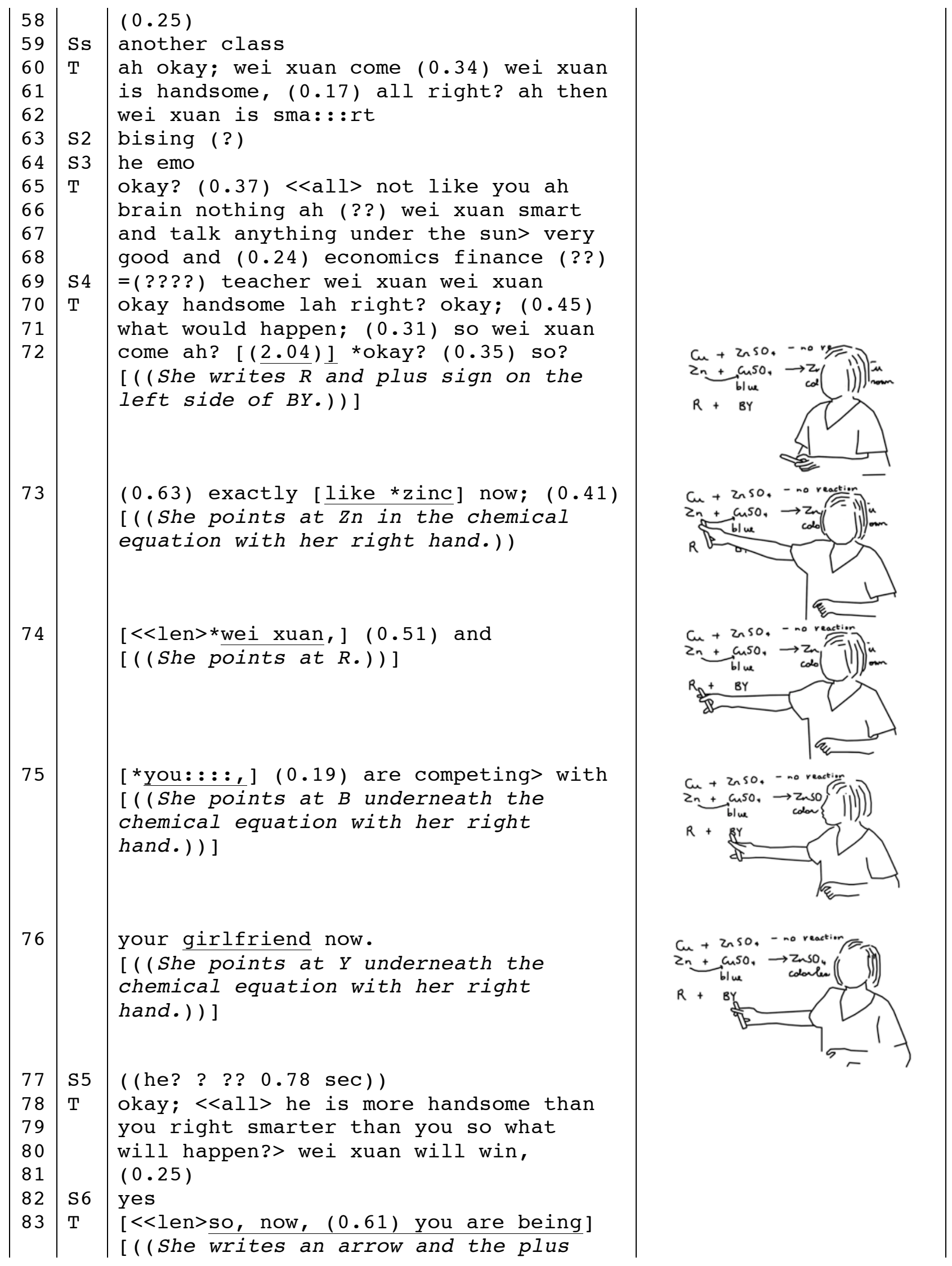




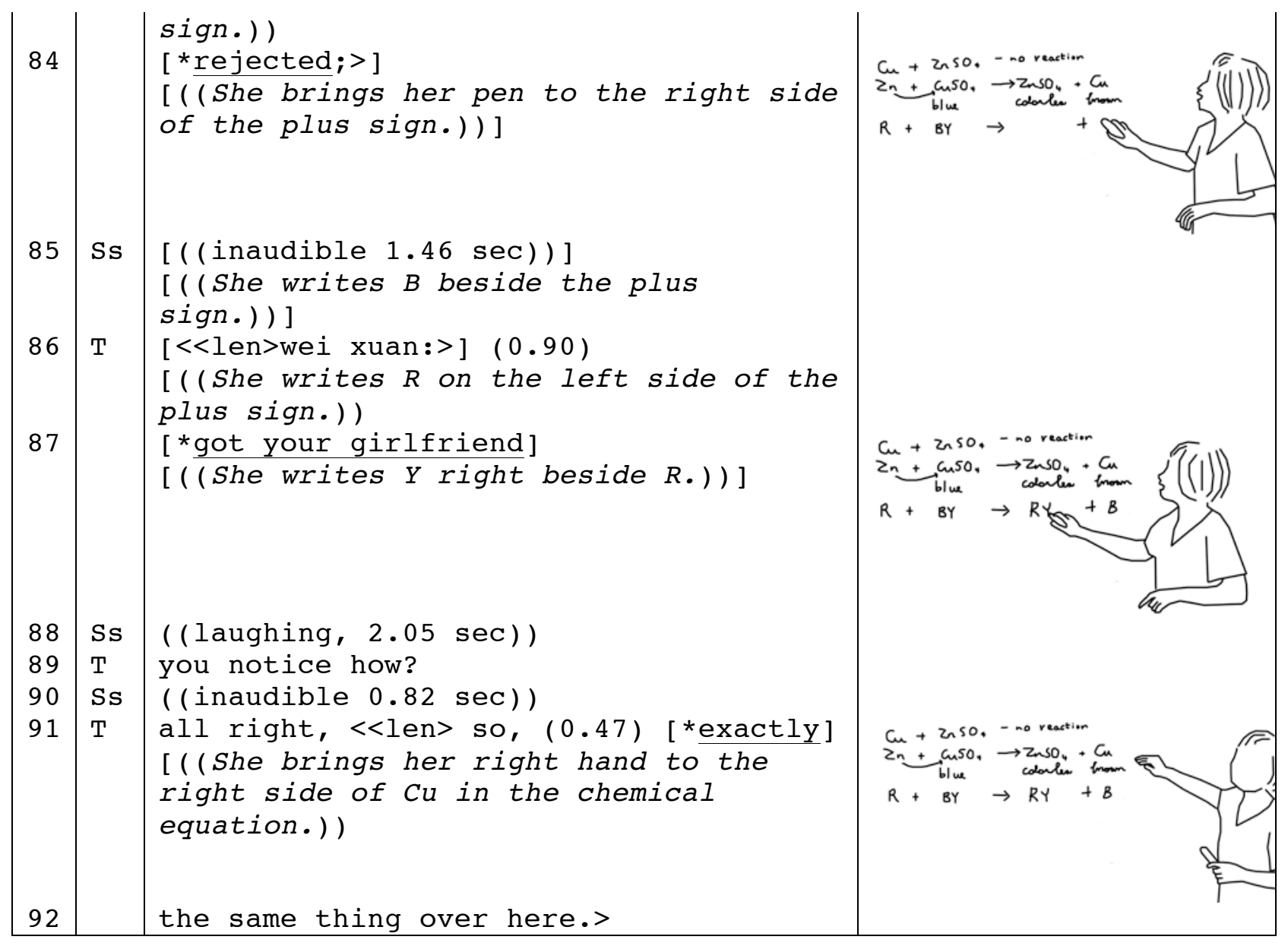




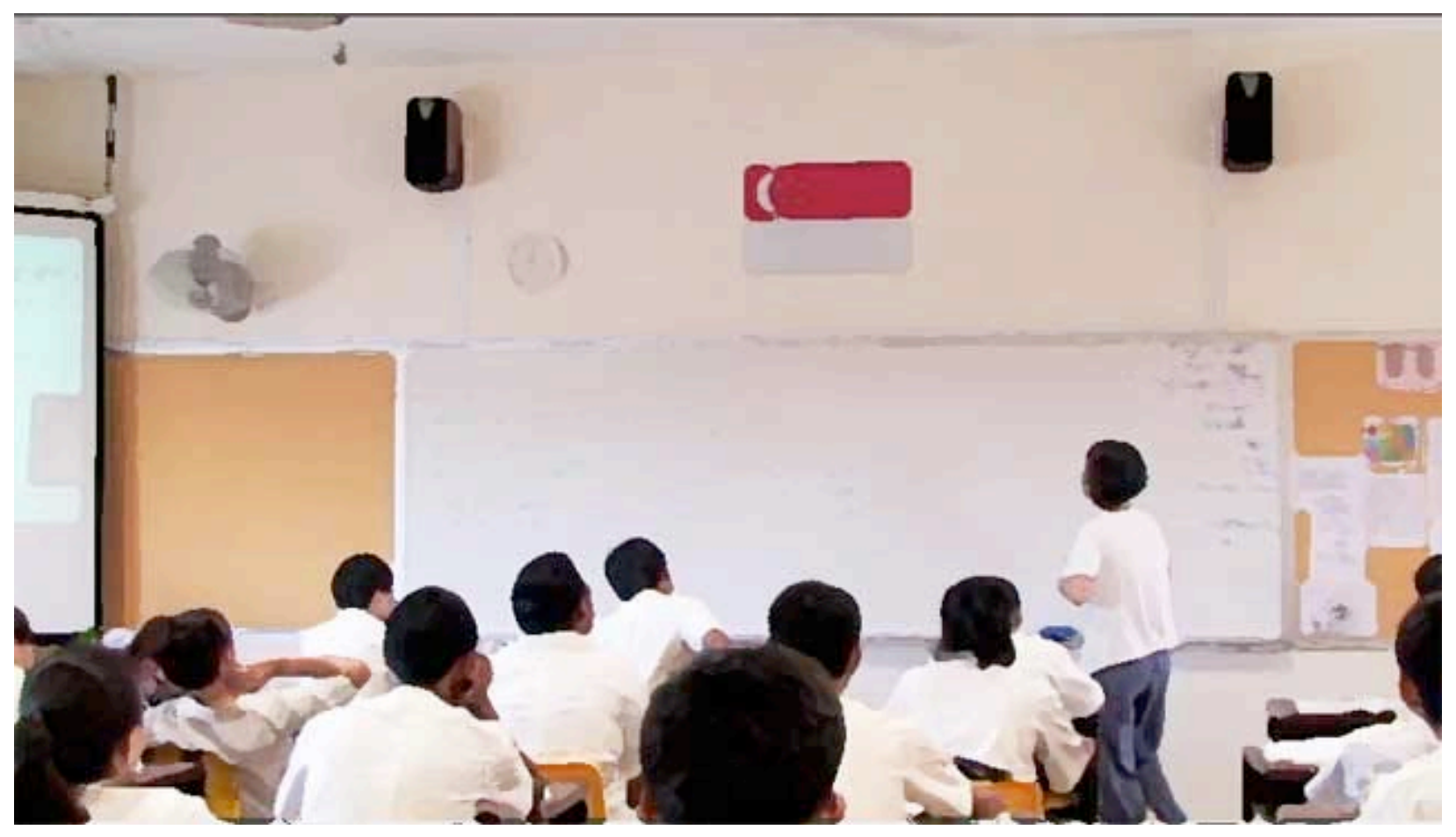

Fig 1. The camera at the back of the classroom mainly followed the chemistry teacher to video record her interaction with students. 


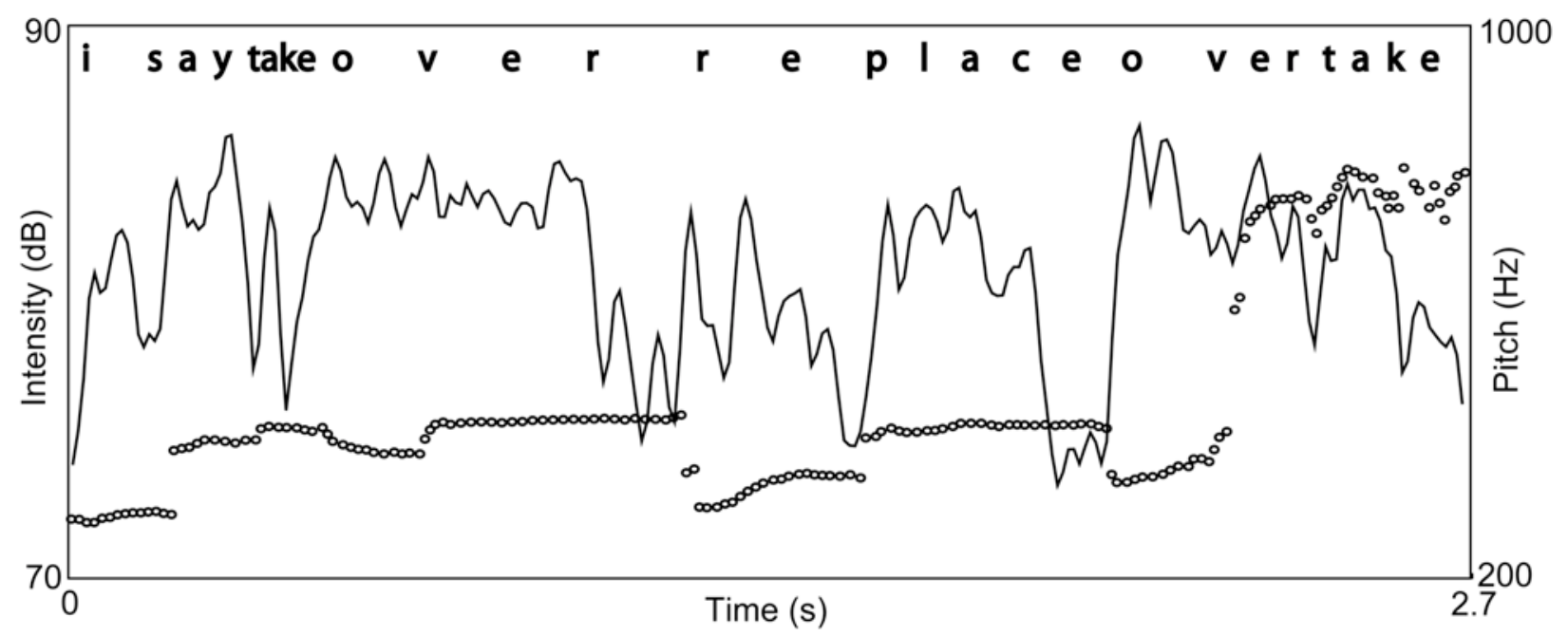

Fig 2. The original video of the case example has been exported to mov and aif formats for subsequent analyses. The speech analysis was conducted with the PRAAT software (www.praat.org). The solid line shows the speech intensity of the utterances (fragment 1 , lines 01-03) by the unit of $\mathrm{dB}$ and the dotted line their pitch contours by the unit of $\mathrm{Hz}$. 


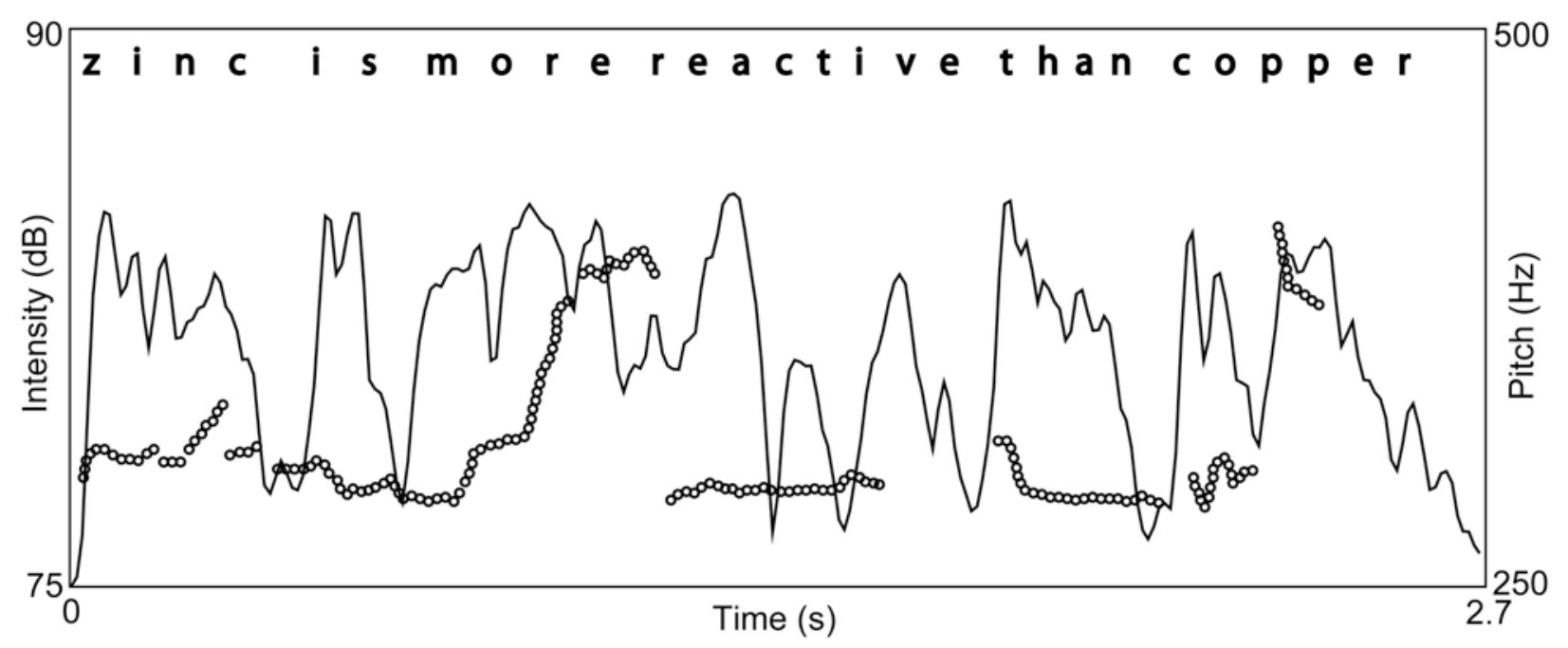

Fig 3. The solid line shows the speech intensity of the utterances (fragment 1, lines 06-08) and the dotted line their pitch contours. 
- deictic gestures

- description of experiment (verbal, and written words e.g., "blue")

- notation on chalkboard

- beat gestures

- prosody (pauses, rate, intensity, pitch, contour)

- talk about description talk

- foreign language talk

Fig 4. Multiple layers of performance 


\section{Chemistry talk $\quad \rightarrow \quad$ Real world talk \\ $\uparrow \quad \uparrow \quad \uparrow$ \\ Chemical equation $\rightarrow \quad$ Invented equation}

Fig 5. Four-part structure of the analogy provided in Fragment 2: two domains (source, target) of analogy are put side by side horizontally and different modalities (i.e., verbal, representations on a board) within each domain are arranged vertically. Each of three types of arrow indicates work for linking different domains and modalities to let them function as an analogy. The horizontal arrows $(\rightarrow)$ between the two domains indicate the corporeal work that is to structure the two verbal talks temporally spread over. The vertical arrows $(\uparrow)$ indicate the work of linking verbal talk and representation. In fragment 2 , the instructor used an invented equation to bring the real world talk into the representational world. The invented equation shows its structural similarity to the chemical equation and takes simultaneous appearance with a verbal stretch of a talk. The linkage of this invented equation to the real world is made through making a diagonal linkage $(\measuredangle)$ between the chemical equation and real world talk. 
Appendix: transcription conventions

\begin{tabular}{|c|c|c|c|}
\hline \multicolumn{2}{|l|}{ Notation } & Description & Example \\
\hline \multicolumn{2}{|c|}{$(0.23)$} & Time without talk, in seconds & overtake; $(0.23)$ so \\
\hline \multicolumn{2}{|l|}{$(? ?)$} & $\begin{array}{l}\text { Question marks in parentheses } \\
\text { indicate (approximate) number of } \\
\text { undecipherable words }\end{array}$ & (0.39) (??) wei xuan \\
\hline \multicolumn{2}{|c|}{$(($ stretches $))$} & $\begin{array}{l}\text { Verbs and descriptions in double } \\
\text { parentheses are transcriber's } \\
\text { comment }\end{array}$ & ((She stretches her right hand)) \\
\hline \multicolumn{2}{|l|}{$::$} & $\begin{array}{l}\text { Colons indicate lengthening of } \\
\text { phoneme, about } 1 / 10 \text { of a second } \\
\text { per colon }\end{array}$ & take ove::::r \\
\hline \multirow{2}{*}{\multicolumn{2}{|c|}{$\left[\begin{array}{l}1 \\
1\end{array}\right.$}} & Square brackets in consecutive & [replace] \\
\hline & & $\begin{array}{l}\text { lines indicate overlap between } \\
\text { words underlined and descriptions } \\
\text { italicized }\end{array}$ & {$[(($ She stretches her right hand $))]$} \\
\hline$<<\mathrm{p}>$ & $>$ & $\begin{array}{l}\text { Piano, lower than normal speech } \\
\text { volume }\end{array}$ & $<<$ p $>$ replace $>$ \\
\hline \multicolumn{2}{|l|}{$\mathrm{SO}$} & $\begin{array}{l}\text { Capital letters indicate emphasized } \\
\text { sounds }\end{array}$ & zinc DISplaces copper \\
\hline$<<$ all $>$ & $>$ & $\begin{array}{l}\text { Allegro, words are uttered with } \\
\text { faster than normal speed }\end{array}$ & $\begin{array}{l}<<\text { all }>\text { competition is between } \\
\text { zinc and copper }>\end{array}$ \\
\hline \multirow{2}{*}{$\begin{array}{l}<<\operatorname{len}> \\
? ?\end{array}$} & $>$ & Lento, slower than normal & $<<$ len $>$ zinc DISplaces copper $>$ \\
\hline & & $\begin{array}{l}\text { Punctuation is used to mark } \\
\text { movement of pitch (intonation) } \\
\text { toward end of utterance, flat, } \\
\text { slightly and strongly upward, and } \\
\text { slightly and strongly downward, } \\
\text { respectively }\end{array}$ & $\begin{array}{l}\text { take over, } \\
\text { is what? } \\
\text { overtake; } \\
\text { happening now. }\end{array}$ \\
\hline \multicolumn{2}{|l|}{$=$} & $\begin{array}{l}\text { Equal sign indicates that the } \\
\text { phonemes of different words are } \\
\text { not clearly separated }\end{array}$ & out of blue?=wei xuan come \\
\hline \multicolumn{2}{|l|}{$*$} & $\begin{array}{l}\text { Asterisk mark denotes an instant } \\
\text { that corresponds to a video- } \\
\text { offprint }\end{array}$ & so exactly; *what \\
\hline
\end{tabular}

